\title{
An Improved Scheme of Wavelength Assignment for Parallel FFT Communication Pattern on a Class of Regular Optical Networks*
}

\author{
Yawen Chen and Hong Shen \\ Japan Advanced Institute of Science and Technology, \\ Asahidai 1-8, Nomi-Shi, Ishikawa, Japan, 923-1292 \\ \{yawen, shen\}@jaist.ac.jp
}

\begin{abstract}
Routing and wavelength assignment (RWA) is a central issue to increase efficiency and reduce cost in Wavelength Division Multiplexing (WDM) optical networks. In this paper, we propose an improved scheme of wavelength assignment of parallel FFT communication pattern on a class of regular optical networks. With our new scheme, the numbers of wavelengths required to realize parallel FFT communication pattern with $2^{n}$ nodes on WDM linear arrays, rings, 2-D meshes and 2-D tori are $\left\lfloor 2^{n-2}+1\right\rfloor,\left\lfloor 2^{n-3}+1\right\rfloor$, $\left\lfloor 2^{\max (k, n-k)-2}+1\right\rfloor$ and $\left\lfloor 2^{\max (k, n-k)-3}+1\right\rfloor$ respectively, which are about one-third less for linear arrays and meshes, and a half less for rings and tori, than the known results. Our results have a clear significance for applications because FFT represents a common communication pattern shared by a large class of scientific and engineering problems and WDM optical networks as a promising technology in networking has an increasing popularity.
\end{abstract}

Keywords: Parallel FFT, wavelength assignment, optical networks, Wavelength Division Multiplexing (WDM), network embedding.

\section{Introduction}

Fast Fourier Transform (FFT) plays an important role in numerous scientific and technical applications [1]. While the application fields of FFT are growing rapidly, the amount of data to be transformed is also increasing tremendously. Hence, there has been a great interest in implementing FFT on parallel computers and some parallel computers have been specially designed to perform FFT computations [2]. With the increasing computation power of parallel computers, interprocessor communication has become an important factor that limits the performance of supercomputing systems. Optical communication, in particular, Wavelength Division Multiplexing (WDM) technique, has become a promising technology for many emerging networking and parallel/distributed computing applications because of its huge bandwidth. Parallel FFT is often implemented on dense interconnection networks such as hypercube and

This work is supported by the $21^{\text {st }}$ Century Center of Excellence Program in JAIST on

"Verifiable and Evolvable e-Society". 
shuffle-exchange networks [2], instead of simple connected networks such as linear arrays and rings. Since WDM divides the bandwidth of an optical fiber into multiple wavelength channels so that multiple devices can transmit on distinct wavelengths through the same fiber concurrently [3], these dense networks can be simplified to simple regular topologies by realizing connections in parallel FFT communication patterns in optical lightpaths. A connection or a lightpath in a WDM network is an ordered pair of nodes $(x, y)$ corresponding to that a packet is sent from source $x$ to destination $y$. In this paper, we assume that no wavelength converter facility is available in the network. Thus, a connection must use the same wavelength throughout its path. Routing and wavelength assignment (RWA) is a key problem for increasing the efficiency of wavelength-routed all-optical networks. RWA can be described as follows [4]: Given a set of all-optical connections, the problem is to (a) find routes from the source nodes to their respective destinations, and (b) assign channels to these routes so that the same channel is assigned to all the links of a particular route. (c) The goal of RWA is to minimize the number of assigned channels. Numerous research studies have been conducted on the RWA problem [3-8]. A popular approach to tackle this problem is to apply integer programming technique, which, however, does not always lead to efficient solution. In [5], the problem of wavelength assignment for realizing parallel FFT communication pattern on a class of regular optical WDM networks was addressed and two methods, sequential mapping and shift-reversal mapping, were proposed. By sequential mapping, the numbers of wavelengths required to realize parallel FFT communication pattern of $2^{n}$ nodes on WDM linear arrays, rings, 2-D meshes and 2-D tori are $2^{n-1}, 2^{n-1}, 2^{\max (k, n-k)-1}$ and $2^{\max (k, n-k)-1}$ respectively. By shift-reversal mapping, the numbers of wavelengths required are $\max \left(3 \times 2^{n-3}, 2\right), 2^{n-2}, \max \left(3 \times 2^{\max (k, n-k)-3}, 2\right)$ and $2^{\max (k, n-k)-2}$ respectively. In this paper, we design a new scheme to realize parallel FFT communication pattern on a class of regular optical WDM networks and results show that our new scheme significantly improves the known results in [5].

\section{Wavelength Assignment of Parallel FFT Communication Pattern}

\subsection{Problem Definition}

The so-called butterfly representation [2] of FFT algorithm is a diagram made up of blocks representing identical computational units (butterflies) connected by arrows that show the flow of data between the blocks. Assuming that $N$ is the length of the sequence to be transformed ( $N$ is an integer power of two), then the diagram with $N\left(\log _{2} N+1\right)$ nodes arranged in $N$ rows and $\log _{2} N+1$ columns is made of $\log _{2} N$ stages of $N / 2$ butterflies each. The butterfly representation clearly shows the great potential of FFT for parallel processing. Generally, the FFT is implemented stage by stage, i.e. any stage of calculation cannot proceed until all the results of its previous stage have been completed. In this paper, we consider one dimensional data sequence of size $N=2^{n}$. If the butterfly representation is viewed as a process graph, i.e. each row of the butterfly is implemented by a process and each arrow by a communication channel, the butterfly can map onto a WDM hypercube perfectly those links connecting the nodes having an 
address that differs by only one bit at each stage. However, if a WDM hypercube is used, only the $i$ th dimensional links are used with one wavelength during the $i$ th stage whereas other $(n-1) \times 2^{n-1}$ links are vacant during this stage, which may lead to wasting of wavelength channels.

As we know, a connection in the hypercube communication pattern is called a dimensional $i$ connection [4] if it connects two nodes that differ in the $i$ th bit position, where $1 \leq i \leq n$. In a network of size $2^{n}$, the set $D I M_{i}$ is defined as the set of all dimension $i$ connections and $H_{n}$ is defined as the hypercube communication pattern which contains all connections in the hypercube. That is, $H_{n}=\bigcup_{i=1}^{n} D I M_{i}$ and $D I M_{i}=\left\{\left(j, j+(-1)^{\left\lfloor j / 2^{n-i}\right\rfloor} \times 2^{n-i}\right) \mid 0 \leq j \leq 2^{n}-1\right\}$. With $2^{n}$ input data distributed on $2^{n}$ processors, the set of all communications during $n$ stages of parallel FFT is equivalent to $H_{n}$, and the set of communications during the $i$ th stage is equivalent to $D I M_{i}$. Clearly, parallel FFT has a regular communication pattern which we denote by $F_{F T}(n \geq 2)$. We model a network as a directed graph $G(V, E)$. Nodes in $V$ are switches and edges in $E$ are links. Since the $n$ stages of parallel FFT communications should be implemented stage by stage, the number of wavelengths required to realize $F F T_{n}$ on optical WDM networks is the maximum number among the wavelengths required by the $n$ stages. Let $W_{e}\left(G^{\prime}, G\right)$ denote the number of wavelengths to realize communication pattern $G^{\prime}$ on network $G$ by embedding scheme $e$. Thus, $W_{e}\left(F F T_{n}, G\right)=\max _{1 \leq i \leq n}\left(W_{e}\left(D I M_{i}, G\right)\right)$.

\subsection{Linear Arrays}

At first, we introduce the definition of cross mapping on linear arrays. Assume that $N_{L}$ and $N_{R}$ are two node arrangements with $2^{n-1}$ nodes numbered from left to right in ascending order starting from 0 . If we put node $i$ of $N_{R}$ between node $2^{n-2}+i$ and node $2^{n-2}+i+1$ of $N_{L}$ for $i=0,1,2, \ldots, 2^{n-2}-2$, and nodes $2^{n-2}-1$ till $2^{n-1}-1$ of $N_{R}$ consecutively after node $2^{n-1}-1$ of $N_{L}$. By symmetry, this is equivalent to placing node $2^{n-2}+i+1$ of $N_{L}$ between node $i$ and node $i+1$ of $N_{R}$, and nodes 0 till $2^{n-2}-2$ of $N_{L}$ consecutively before node 0 of $N_{R}$. We call the above operation cross operation and denote the obtained node arrangement $\operatorname{Cross}\left(N_{L}, N_{R}\right)$. Assume that $X_{n}$ is the increasing order of indices in binary representations of $2^{n}$ nodes. For example, $X_{2}=00,01,10,11$. We define the cross order of a linear arrays with $2^{n}$ nodes, $C_{n}(n \geq 1)$, as follows: $C_{n}=\left\{\begin{array}{l}X_{n}, n \leq 2 \\ \operatorname{Cross}\left(0 X_{n-1}, 1 X_{n-1}\right), n \geq 3\end{array} \quad\right.$. For example, $C_{4}=\operatorname{Cross}\left(0 X_{3}, 1 X_{3}\right)=$ Cross ((0000, 0001, 0010, 0011, 0100, 0101, 0110, 0111), (1000, 1001, 1010, 1011, 1100, $1101,1110,1111))=0000,0001,0010,0011,0100,1000,0101,1001,0110,1010$, 0111, 1011, 1100, 1101, 1110, 1111. 
Assume that the nodes of WDM linear arrays are numbered from left to right in ascending order starting from 0 , and that the links are numbered from left to right starting from 1 . If the $i$ th node of $\mathrm{C}_{n}$ for $F F T_{n}$ is mapped onto the $i$ th processor of the WDM network $G$, we establish the 1-1 mapping from the nodes of $F F T_{n}$ to the nodes of $G$. We define such an embedding cross mapping on WDM linear arrays. Figure 1 shows cross mapping of $F F T_{3}$ on 8-node linear array.

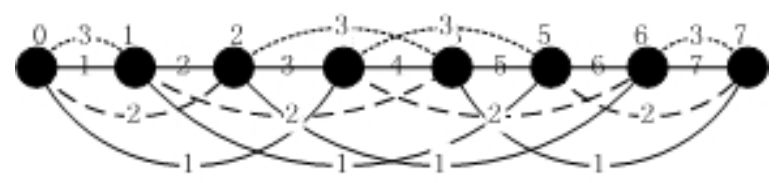

Fig. 1. Cross mapping on 8-node linear array

Theorem 1. By cross mapping, the number of wavelengths required to realize $F F T_{n}$ on an $2^{n}$-node WDM linear array is $\left\lfloor 2^{n-2}+1\right\rfloor$.

Proof. When $n=1$ and 2, it is easy to know the results are true. In the following, we consider the numbers of wavelengths required during the $n$ stages when $n \geq 3$.

When $n \geq 3$, the $i$ th node of $N_{L}\left(0 X_{n-1}\right)$ communicates with the $i$ th node of $N_{R}$ ( $1 X_{n-1}$ ) during the first stage. Assuming that the number of nodes in $N_{L}$ on the left side of the $i$ th link on the linear array is $l_{i}^{l}$ by cross mapping, and the number of nodes in $N_{R}$ on the left side of the $i$ th link is $r_{i}^{l}$, then the number of wavelengths required on the $i$ th link during the first stage is $l_{i}^{l}-r_{i}^{l}$. It can be calculated that the number of wavelengths required during the first stage on the $i$ th link, denoted by $w_{i 1}$, is $w_{i 1}=\left\{\begin{array}{l}i, 1 \leq i \leq 2^{n-2} \\ 2^{n-2}, 2^{n-2}+1 \leq i \leq 3 \times 2^{n-2}-1 \text { and } i \text { is even } \\ 2^{n-2}+1,2^{n-2}+1 \leq i \leq 3 \times 2^{n-2}-1 \text { and } i \text { is odd } \\ 2^{\mathrm{n}}-\mathrm{i}, 3 \times 2^{n-2} \leq i \leq 2^{n}-1\end{array}\right.$. Therefore, the number of wavelengths required during the first stage is $2^{n-2}+1$. During the second stage, there is no communications between the nodes of $N_{L}$ and $N_{R}$. If the cross operation is not implemented, communications within nodes of $N_{L}$ and nodes of $N_{R}$ are equivalent to the communications of $F F T_{n-1}$ mapped on $2^{n-1}$-node linear arrays by sequential mapping [5]. So, the number of wavelengths required on the $i$ th link of the two $2^{n-1}$-node linear arrays is $i$ for $1 \leq i \leq 2^{n-2}-1$ and $2^{n-1}-i$ for $2^{n-2} \leq i \leq 2^{n-1}-1$. If the cross operation is implemented between these two $2^{n-1}$-node linear arrays, the relative positions between the nodes within $N_{L}$ and $N_{R}$ are not changed and the number of wavelengths required on each link is the sum of wavelengths required on the corresponding links 
which are overlapped between the two $2^{n-1}$-node linear arrays. Assuming that the number of nodes in $N_{L}$ on the right side of the $i$ th $\left(2^{n-2}+1 \leq i \leq 3 \times 2^{n-2}-1\right)$ link on linear arrays is $l_{i}^{r}$ by cross mapping and the number of nodes in $N_{R}$ on the left side of the $i$ th link is $r_{i}^{l}$, the number of wavelengths required on the $i$ th link during the second stage is $l_{i}^{r}+r_{i}^{l}$. It can be calculated that the number of wavelengths required during the second stage on the $i$ th link, denoted by $w_{i 2}$, is $w_{i 2}=\left\{\begin{array}{l}i, 1 \leq i \leq 2^{n-2} \\ 2^{n-2}, 2^{n-2}+1 \leq i \leq 3 \times 2^{n-2}-1 \text { and } i \text { is even } \\ 2^{n-2}-1,2^{n-2}+1 \leq i \leq 3 \times 2^{n-2}-1 \text { and } i \text { is odd } \\ 2^{n}-i, 3 \times 2^{n-2} \leq i \leq 2^{n}-1\end{array}\right.$. Therefore, the number of wavelengths required during the second stage is $2^{n-2}$.

During the $j$ th stage for $3 \leq j \leq n$, the number of wavelengths required is $2^{n-j}$ on each of $N_{L}$ and $N_{R}$ before the cross operation [5]. After the cross operation, the numbers of wavelengths required during stage from 3 to $n$ are less than $2 \times 2^{n-j} \leq 2 \times 2^{n-3}=2^{n-2}$ because the number of wavelengths required on the $2^{n}$-node linear array is not more than the double of the wavelengths required on each of $N_{L}$ and $N_{R}$ in the worst case. Therefore, the maximum number of wavelengths required during all stages by cross mapping is $\left\lfloor 2^{n-2}+1\right\rfloor$.

Clearly, realizing $F F T_{n}$ on an $2^{n}$-node WDM linear array by cross mapping requires $2^{n-3}-1$ fewer wavelengths than that by shift-reversal mapping mentioned in [5] when $n \geq 4$.

\subsection{Rings}

If we exchange node $i$ of $N_{L}$ with node $2^{n-1}-i$ of $N_{R}$ and exchange node $2^{n-1}-i$ of $N_{L}$ with node $i$ of $N_{R}$ for each $i=1,3,5, \ldots, 2^{n-3}-1$, we call such an operation exchange operation and denote the obtained node arrangement Exchange $\left(N_{L}, N_{R}\right)$. Assume that $C^{-1}$ is the reversal arrangement of $C$. For example, if $C=a, b, c, d$, then $C^{-1}=d, c, b, a$. Thus, we define the cross order on rings, denoted by $C R_{n}(n \geq 1)$ as follows: $C R_{n}=\left\{\begin{array}{l}0 C_{n-1}, 1 C_{n-1}^{-1}, n \leq 3 \\ \text { Exchange }\left(0 C_{n-1}, 1 C_{n-1}^{-1}\right), n \geq 4\end{array}\right.$. For example, $C R_{4}=\operatorname{Exchange}\left(0 C_{3}, 1 C_{3}^{-1}\right)=$ Exchange (0(000, 001, 010, 100, 011, 101, 110, 111), 1(000,001,010,100,011, $\left.101,110,111)^{-1}\right)=0000,1000,0010,0100,0011,0101,0110,1110,1111,0111,1101$, $1011,1100,1010,1001,0001$. Assume that the nodes of WDM rings are numbered clockwise starting from 0 , and the links starting from 1 . If we map the $i$ th node on $C R_{n}$ of $F F T_{n}$ onto the $i$ th processor of WDM rings, we establish the 1-1 mapping from the 
nodes of $\mathrm{FFT}_{n}$ to the nodes of rings. We define such an embedding cross mapping on rings.

Theorem 2. By cross mapping, the number of wavelengths required to realize $F F T_{n}$ on an $2^{n}$-node WDM ring is $\left\lfloor 2^{n-3}+1\right\rfloor$.

Proof. It is easy to know the numbers of wavelengths required on the rings for $n=1,2$ and 3 are 1, 1, and 2 respectively. In the following, we consider the numbers of wavelengths required during the $n$ stages when $n \geq 4$.

During the first stage, the $i$ th node of $N_{L}$ communicates with node $2^{n-1}-1-i$ of $N_{R}$. Exchange operation results that the $i$ th node on the ring communicates with node $i+1$ for $i \in\left\{2 k \mid 0 \leq k \leq 2^{n-4}-1,3 \times 2^{n-4} \leq k \leq 5 \times 2^{n-4}-1,7 \times 2^{n-4} \leq k \leq 2^{n-1}-1\right\}$. As those communications take place between the neighborhood nodes, the number of wavelengths required is 1 . At the same time, the $i$ th node on the ring communicates with node $2^{n-1}-i$ for $2^{n-3} \leq i \leq 3 \times 2^{n-3}-1$, which requires $2^{n-3}$ wavelengths. Therefore, the number of wavelengths required during the first stage is $2^{n-3}+1$.

During the stages from 2 to $n$, there is no communications passing through the links of $2^{n-1}$ and $2^{n}$ if the exchange operation is not implemented. If we ignore these two links, the ring can be regarded as two $2^{n-1}$-node linear arrays. By the definition of cross mapping on rings, realizing the stages from 2 to $n$ can be regarded as realizing $F F T_{n-1}$ on each $2^{n-1}$-node linear array by cross mapping before the exchange operation, which requires $2^{n-3}+1$ wavelengths by Theorem 1 . In the following, we prove that the number of wavelengths is still $2^{n-3}+1$ after exchange operation.

Due to the symmetry of the ring, the numbers of wavelengths required on the links clockwise from $7 \times 2^{n-3}$ to $2^{n}$ and 1 to $2^{n-3}$ are equal with those on the links clockwise from $3 \times 2^{n-3}$ to $5 \times 2^{n-3}$. So, we only take the links from $7 \times 2^{n-3}$ to $2^{n}$ and 1 to $2^{n-3}$ for example. Before the exchange operation, the maximum number of wavelengths required on the $i$ th $\left(1 \leq i \leq 2^{n-3}, 7 \times 2^{n-3} \leq i \leq 2^{n}\right)$ link of the ring, denoted by $w_{i}$, satisfies $w_{i} \leq\left\{\begin{array}{l}i, 1 \leq i \leq 2^{n-3} \\ 2^{\mathrm{n}}-i, 7 \times 2^{n-3} \leq i \leq 2^{n}\end{array}\right.$. After the exchange operation, the exchange between node $i$ and node $2^{n}-i$ results that the numbers of wavelengths required on the links clockwise from $2^{n}-i$ to $2^{n}$ and from 1 to $i$ increase by 1 for each $i=1,3,5, \ldots, 2^{n-3}-1$ in the worst case. So, the number of additional wavelengths passing through the $i$ th link caused by the exchange operation, denoted by $\Delta w_{i}$, satisfies $\Delta w_{i} \leq\left\{\begin{array}{l}2^{n-3}-i, 1 \leq i \leq 2^{n-3} \text { and } i \text { is even } \\ 2^{n-3}-i-1,1 \leq i \leq 2^{n-3} \text { and } i \text { is odd } \\ i-7 \times 2^{n-3}, 7 \times 2^{n-3} \leq i \leq 2^{n} \text { and } i \text { is even } \\ i-7 \times 2^{n-3}-1,7 \times 2^{n-3} \leq i \leq 2^{n} \text { and } i \text { is odd }\end{array}\right.$. Therefore, the maximum number of wavelengths required on the $i$ th links is not more than 


$$
w_{i}+\Delta w_{i} \leq\left\{\begin{array}{l}
2^{n-3}, 1 \leq i \leq 2^{n-3} \text { and } i \text { is even } \\
2^{n-3}-1,1 \leq i \leq 2^{n-3} \text { and } i \text { is odd } \\
2^{n-3}, 7 \times 2^{n-3} \leq i \leq 2^{n} \text { and } i \text { is even } \\
2^{n-3}-1,7 \times 2^{n-3} \leq i \leq 2^{n} \text { and } i \text { is odd }
\end{array}\right. \text {. It can be concluded that the }
$$

maximum number of wavelengths required on the links clockwise from $7 \times 2^{n-3}$ to $2^{n}$ and 1 to $2^{n-3}$ is not more than $2^{n-3}+1$. Therefore, the number of wavelengths required on WDM rings with $2^{n}$ nodes is $\left\lfloor 2^{n-3}+1\right\rfloor$. From the above discussion, we know that the wavelengths required to realize $F F T_{n}$ in WDM ring with $2^{n}$ nodes by cross mapping is $2^{n-3}-1$ less wavelengths than that by shift-reversal mapping when $n \geq 4$.

We denote sizes of meshes and tori as $N=2^{k} \times 2^{n-k}$. For simplicity, the details of the definition for the cross mapping on meshes and tori are ignored here.

Theorem 3. By cross mapping, the numbers of wavelengths required to realize $F F T_{n}$ on a $2^{k} \times 2^{n-k}$ mesh and torus are $\left\lfloor 2^{\max (k, n-k)-2}+1\right\rfloor$ and $\left\lfloor 2^{\max (k, n-k)-3}+1\right\rfloor$ respectively.

\section{Comparisons}

It can be seen that cross mapping outperforms shift-reversal mapping and sequential mapping on the number of wavelengths, as shown in Fig. 2 for linear arrays. The analysis can be obtained similarly for other topologies.

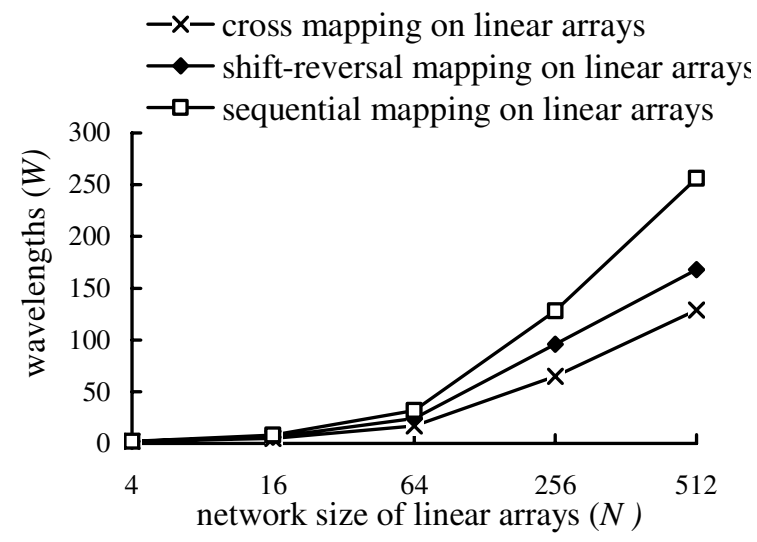

Fig. 2. Comparisons of wavelengths required on liner arrays 


\section{Conclusions}

In this paper, we proposed an improved scheme of wavelength assignment for parallel FFT communication pattern on a class of regular optical networks. By the improved mapping method, the numbers of wavelengths required to realize parallel FFT communication pattern with $2^{n}$ nodes on WDM linear arrays, rings, 2-D meshes and $2-\mathrm{D}$ tori are $\left\lfloor 2^{n-2}+1\right\rfloor,\left\lfloor 2^{n-3}+1\right\rfloor,\left\lfloor 2^{\max (k, n-k)-2}+1\right\rfloor$ and $\left\lfloor 2^{\max (k, n-k)-3}+1\right\rfloor$ respectively, which improved the results in [5]. Our results have a clear significance for applications because FFT represents a common communication pattern shared by a large class of scientific and engineering problems and WDM optical networks as a promising technology in networking has an increasing popularity. Future work may include other type of optical networks and other RWA problems. Another interesting issue is to find the lower bound for this problem and the improved schemes which can achieve the lower bound.

\section{References}

1. Rami A, AL-Na'mneh, W. David Pan, and B. Earl Wells. Two parallel implementations for one dimension FFT on symmetric multiprocessors. ACM Southeast Regional Conference, pp. 273-278. ACM Press. New York, NY, USA, 2004.

2. F. T. Leighton. Introduction to Parallel Algorithms and Architectures: Arrays, Trees, Hypercubes. Morgan Kaufmann Publishers, Inc., 1992.

3. Hui Zang, Jason P. Jue, and Biswanath Mukherjee. A review of routing and wavelength assignment approaches for wavelength-routed optical WDM networks. SPIE Optical Networks Magazine, 1(1):47-60, 2000.

4. Yuan X and Melhem R. Optimal Routing and Channel Assignments for Hypercube Communication on Optical Mesh-like Processor Arrays. Proceedings of the 5th International Conference on Massively Parallel Processing Using Optical Interconnection, pp.110-118. IEEE Computer Society Press. Las Vegas, NV, 1998.

5. Fangai Liu and Yawen Chen. Wavelength Assignment of Parallel FFT Communication Pattern in a Class of Regular Optical WDM Network. Proceedings of the IEEE International Symposium on Parallel Architectures, Algorithms, and Networks, 495-500. IEEE Computer Society. Hong Kong, 2004.

6. Zhou Chunling and Yang Yuanyuan. Wide-Sense nonblocking multicast in a class of regular optical WDM networks. IEEE Transactions on Communications, 50(1):126-134, 2002.

7. H. Shen, Y. Pan, J. Sum and S. Horiguchi, Multicasting in multihop optical WDM networks with limited wavelength conversion. IEICE Transactions on Information and Systems, E86-D(1):3-14, 2003.

8. Yawen Chen and Fangai Liu. A Wavelength Assignment Algorithm of Parallel LU Decomposition Communication Pattern On WDM Ring Interconnection Network. International Symposium on Distributed Computing and Applications to Business, Engineering and Science, pp. 366-370. Wuhan, China, 2004. 